\title{
Construction and validation of nomograms for non-metastatic Ewing sarcoma: A prognostic factor analysis based on the SEER database
}

\author{
RUNZHI HUANG ${ }^{1-3^{*}}$, DONG HAN ${ }^{1 *}$, CHENGCHENG SHI $^{1 *}$, PENGHUI YAN $^{1}$, PENG HU $^{1}$, \\ XIAOLONG ZHU ${ }^{1}$, HUABIN YIN ${ }^{4}$, TONG MENG ${ }^{2,3}$ and ZONGQIANG HUANG ${ }^{1}$
}

\author{
${ }^{1}$ Department of Orthopaedics, The First Affiliated Hospital of Zhengzhou University, Zhengzhou, Henan 450052; \\ ${ }^{2}$ Division of Spine Surgery, Department of Orthopaedics, Tongji Hospital, Tongji University School of Medicine; \\ ${ }^{3}$ Key Laboratory of Spine and Spinal Cord Injury Repair and Regeneration (Tongji University), Ministry of Education, \\ Shanghai 200065; ${ }^{4}$ Department of Orthopaedics, Shanghai Bone Tumor Institute, Shanghai General Hospital, \\ School of Medicine, Shanghai Jiaotong University, Shanghai 200080, P.R. China
}

Received August 14, 2019; Accepted December 18, 2020

DOI: $10.3892 / 01.2021 .13038$

\begin{abstract}
Ewing sarcoma is the second most common osseous disease in children and adolescents. It presents with a poor prognosis due to the high degree of malignancy and distant metastasis. In order to predict the disease prognosis and investigate a suitable therapeutic strategy for Ewing sarcoma, the present study aimed to describe the clinical characteristics, and to construct and validate nomograms for patients with non-metastatic Ewing sarcoma. A total of 627 cases of non-metastatic Ewing sarcoma were retrospectively collected from the Surveillance, Epidemiology, and End Results database between 2005 and 2014. Survival analysis and a machine learning model were used to identify independent prognostic variables and establish nomograms to estimate overall survival (OS) and cause-specific survival (CSS). The
\end{abstract}

Correspondence to: Professor Tong Meng, Division of Spine Surgery, Department of Orthopaedics, Tongji Hospital, Tongji University School of Medicine, 389 Xincun Road, Shanghai 200065, P.R. China

E-mail: mengtong@medmail.com.cn

Professor Zongqiang Huang, Department of Orthopaedics, The First Affiliated Hospital of Zhengzhou University, 1 Jianshe Road, Zhengzhou, Henan 450052, P.R. China

E-mail: gzhuangzq@163.com

${ }^{*}$ Contributed equally

Abbreviations: OS, overall survival; CSS, cause-specific survival; SEER, Surveillance, Epidemiology, and End Results; AJCC, American Joint Committee on Cancer; ICD.O.3, International Classification of Diseases for Oncology, 3rd Edition; MDG, Mean Decrease Gini; HR, hazard ratio; CI, confidence interval

Key words: non-metastatic Ewing sarcoma, prognostic factor, survival analysis, nomogram, external validation nomograms were bootstrap internally validated and externally validated using non-metastatic Ewing sarcoma cases from the First Affiliated Hospital of Zhengzhou University. The accuracy was also assessed by comparing with current American Joint Committee on Cancer (AJCC) staging systems. The total series consisted of 627 patients with non-metastatic Ewing sarcoma with a mean age of 20.14 years. Age, tumor extension, sex, International Classification of Diseases for Oncology, 3rd Edition histology, surgery and chemotherapy were identified as independent risk factors for OS and CSS. The aforementioned outcomes were incorporated to construct the nomograms, and the concordance indices (C-indices) for internal validation of OS and CSS prediction were 0.791 and 0.813 , which were higher than those for AJCC sixth edition (OS, 0.531; CSS, 0.534 ) and seventh edition (OS, 0.547; CSS, 0.561), while the $\mathrm{C}$-indices for external validation of OS and CSS prediction were 0.834 and 0.825 , respectively. In conclusion, age, sex, tumor extension and surgery were independent prognostic factors for both OS and CSS. In addition, with regard to OS, the Ewing sarcoma subtype was a poor factor and chemotherapy was a favorable one. Nomograms based on reduced Cox models attained a satisfactory accuracy in predicting the survival of patients with non-metastatic Ewing sarcoma and could assist clinicians in evaluating survival more accurately.

\section{Introduction}

Ewing sarcoma is the second most common osseous disease in children and adolescents, and predominantly occurs in the pelvic bones, femur and chest wall $(1,2)$. Pathologically, it stems from primitive neuroepithelial cells, which are small round cells with high expression levels of CD99 with the potential to differentiate into various types of mesenchymal cell $(1,3,4)$. Ewing sarcoma is characterized by the fusion of the EWS gene on chromosome 22q12 (1).

Ewing sarcoma is treated with adjuvant chemotherapy following wide excision or amputation with or without radiotherapy, as determined by the National Comprehensive 
Cancer Network (NCCN) guidelines $(5,6)$. Despite various treatment strategies, the 5-year-overall survival (OS) rate is reported to have been only $65 \%$ in Turkey between 2001 and 2010, which may be associated with the high metastatic rate noted at an early stage (7). In addition, metastases are often regarded as the most significant predictor of OS in patients with Ewing sarcoma $(8,9)$. Other potential factors include primary tumor location, tumor volume and lactate dehydrogenase levels $(10,11)$. Non-metastatic Ewing sarcoma exhibits relatively mild symptoms and favorable prognosis compared with the metastatic type of the disease (8). Therefore, the description of its clinical characteristics and the investigation into its prognostic factors can assist clinical decision-making.

With regard to non-metastatic Ewing sarcoma, surgery is more useful than chemotherapy due to the high probability of en bloc tumor resection $(8,10)$. Chemotherapy is the main adjuvant treatment method for Ewing sarcoma and was used in $\sim 92 \%$ of non-metastatic Ewing sarcoma in previous studies $(11,12)$. Furthermore, it has been demonstrated to be a favorable predictor for OS (7-12). Chemotherapy decreases the proliferation rate of the remaining tumor cells and improves patient survival time (1). With regard to radiotherapy, it has also been reported that chemotherapy is an appropriate regimen for local control in patients with Ewing sarcoma (13).

A nomogram is a visual, statistical predictive tool that has been designed to identify prognostic factors and predict disease-specific prognosis for each patient. Its predictive accuracy is superior to that of transitional prognostic scoring systems, such as the American Joint Committee on Cancer (AJCC) Tumor-Node-Metastasis staging system (6). However, to the best of our knowledge, the application of nomograms in the prediction of the prognosis for patients with non-metastatic Ewing sarcoma has not been previously investigated. In the present study, a machine learning model (random forest) and survival analysis methods, including the Kaplan-Meier (K-M) curve and Cox proportional hazards regression model, were used to identify independent prognostic variables for non-metastatic Ewing sarcoma from large sample data derived from publicly available sources [Surveillance, Epidemiology and End Results (SEER) database]. Subsequently, nomograms were constructed to estimate OS and cause-specific survival (CSS). Furthermore, external validation was performed to evaluate the accuracy and reproducibility of the nomograms in clinical applications.

\section{Materials and methods}

Patient selection. The present study was approved by the Ethics Committee of The First Affiliated Hospital of Zhengzhou University (no. KEYAN-2018-LW-023; Zhengzhou, China). The data analyzed in the program were identified between January 2005 and December 2014 from the SEER database (https://seer.cancer.gov/). Only patients diagnosed with Ewing sarcoma as the primary malignancy were included. Patients with tumors that were not first tumors (patients with no prior history of tumors or patients with tumors that were not primary tumors) were excluded from the present study. The tumor size data were collected with the exception of the codes 000/888/999/989-998 ('000' indicated no mass or no tumor found; ' 888 ' and '999' indicated not applicable or unknown; '989-998' indicated $989 \mathrm{~mm}$ or larger but not accurately recorded). The tumor extension data were also collected with the exception of the codes 000/888/999/989-998. The exclusion criteria were as follows: Patients without biopsy diagnosis, patients with the NOM0 stage and patients who did not have Ewing sarcoma as their primary malignancy, patients with unknown surgical information, and patients with unknown marital status and ethnicity. Several studies have reported that marital status is an important prognostic factor for various tumor types $(14,15)$. Therefore, in order to include marital status in the subsequent analysis, subjects with unknown marital status were removed. The patient selection is shown in the flow chart in Fig. 1. Patient characteristics and the original dataset are available in Tables SI and SII, respectively.

A cohort was formed for validation according to the AJCC 6 and 7th edition guidelines using the same exclusion criteria from the SEER database [this was a cohort with only AJCC stage as the independent variables and survival data as the dependent variable from the same SEER database using SEER*STAT (version 8.3.6; seer.cancer.gov/seerstat), and all patients with unknown AJCC stage were excluded from this cohort] (6). In addition, non-metastatic Ewing sarcoma cases between January 2013 and December 2018 at The First Affiliated Hospital of Zhengzhou University (Zhengzhou, China) were collected for external validation using the Electronic Medical Record System using the same inclusion and exclusion criteria. Despite the rarity of the disease, an external dataset comprising 26 cases was finally collected. Patient characteristics are shown in Table SIII.

Data extraction. The variables analyzed in the present study included the baseline demographic features of the patients (age at diagnosis, sex and ethnicity), the tumor characteristics [size, extension, primary site, International Classification of Diseases for Oncology, 3rd Edition (ICD.O.3) histological subtype and AJCC T stage] $(6,16)$, the medical treatment (surgery, radiation recode and chemotherapy recode) and the socioeconomic variables (marital status, degree of education, family income and employment status). Age was divided into three subgroups, namely children $(<15$ years), adolescents and young adults (15-39 years), and old adults ( $>39$ years) according to the NCCN Guidelines for Adolescent and Young Adult Oncology (17). The endpoint of the present study was patient death, which was presented as OS and CSS. The follow-up endpoint of all patients was December 31, 2014.

Statistical analysis. The categorical variables were expressed as percentages and the continuous variables were presented as the median (range). Subsequently, the continuous variables were transformed into categorical variables, such as age, tumor size and tumor extension. According to the NCCN guidelines (version 2017) (6), age $(<15,15-39$ and $>39$ years), tumor size $(<80$ and $\geq 80 \mathrm{~mm})$ and tumor extension $(<300$ and $\geq 300 \mathrm{~mm})$ were divided into different subgroups. In addition, patients were divided into subgroups according to the median values for socioeconomic variables. The $\chi^{2}$ test was adopted to assess the distinction between the sub-variables of each categorical variable. The same processes were performed based on OS 


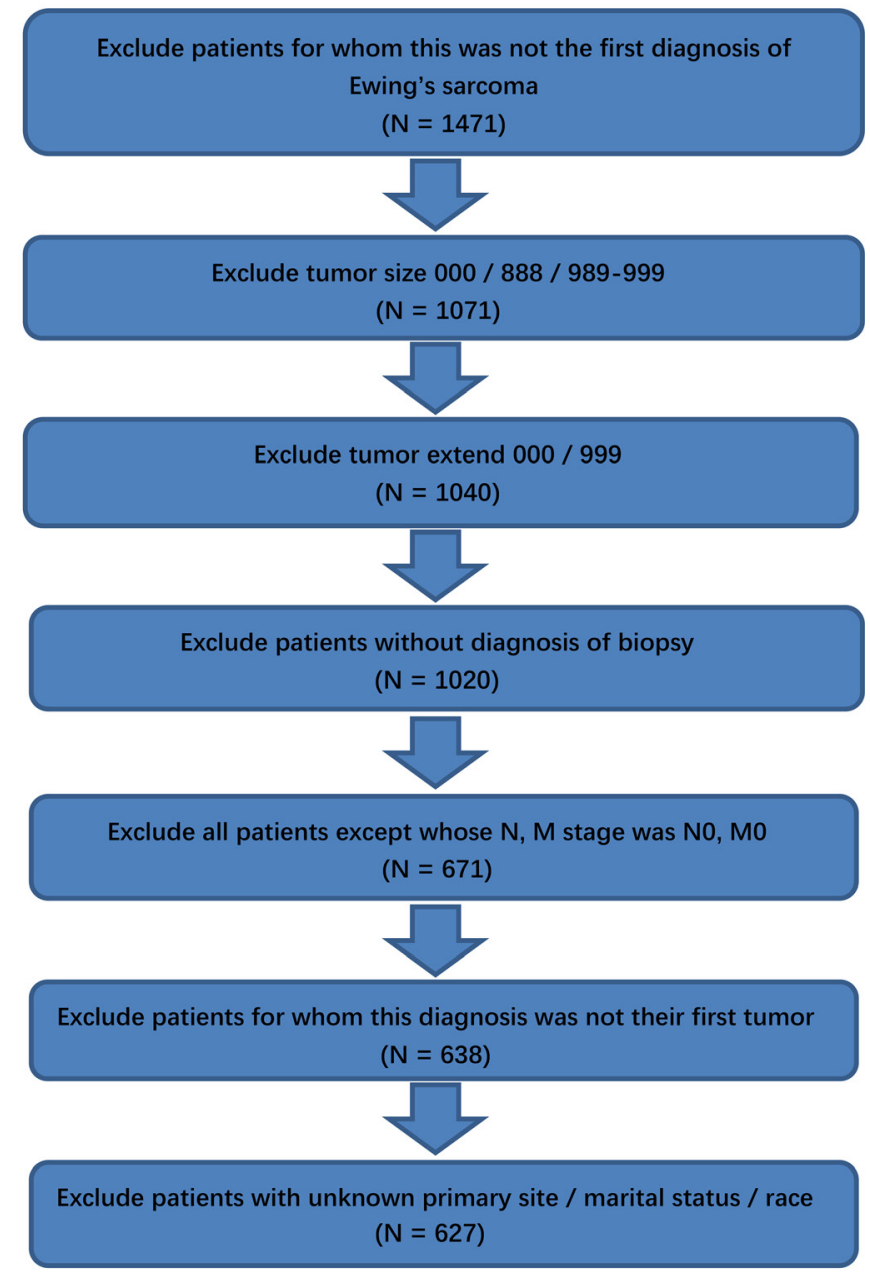

Figure 1. Flow chart for creation of the Surveillance, Epidemiology, and End Results dataset.

and CSS. To compare the OS and CSS between subtypes, a $\mathrm{K}-\mathrm{M}$ curve was used. For the K-M survival analysis, a log-rank test was used to compare the survival curves of each variable.

A random forest plot was established for entire variables. The Mean Decrease Gini (MDG) was used to estimate how each variable contributed to the OS and CSS. Additionally, the out-of-bag error was used to assess the accuracy of the classification in the random forest. The Cox proportional hazards model was constructed based on the results of the univariate analysis. The significant variables of the univariate analysis were screened to structure the subsets used as the data of the full Cox model. The non-significant chemotherapy effects were also retained for subsequent analysis. As a result, a reduced Cox model was constructed by sorting out the significant predictors from the full Cox model. Although the surgical information was not significant in the full Cox model, it was reserved for subsequent analysis in the reduced Cox model. Following double selections, the Cox model with the optimum predictors was eventually selected.

Based on the reduced Cox model, the nomograms were constructed for the evaluation of OS and CSS. The discriminations between predicted and observed values were evaluated via the concordance index (C-index) of internal validation. Their calibration was subsequently estimated using the corresponding calibration curve. In addition, to assess the accuracy of the final models, the external validation and concordance of the two AJCC editions was performed as the last step.

In the present study, R version 3.5.0 software (https:// www.r-project.org/) was used to identify the statistically significant variables with a two-sided $\mathrm{P}<0.05(\alpha=0.05)$. The R packages ggplot2 (version 3.3.2; https://cloud.rproject.org/web/packages/ggplot2/index.html), survminer (version 0.4.8; https://cloud.r-project.org/web/packages/ survminer/index.html), survival (version 3.2-7; https:// cloud.r-project.org/web/packages/survival/index.html), rms (version 6.0-1; https://cloud.r-project.org/web/packages/rms/ index.html) and randomForest (version 4.6-14; https://cloud.rproject.org/web/packages/randomForest/index.html) were used to establish the model and draw the curves, as well as the nomograms.

\section{Results}

Baseline characteristics. A total of 1,471 cases from the SEER database were selected between 2005 and 2014 as primary malignant cases. These cases were filtered, and an entire cohort containing 627 patients was included based on the inclusion and exclusion criteria. The patient characteristics are listed in Table SI, and the raw dataset of the entire primary cohort comprising 1,471 patients with non-metastatic Ewing sarcoma is shown in Table SII. At the end of the follow-up period, 502 patients were alive and 125 patients did not survive. The median age was 17 years (range, 0-85 years) and the highest proportion was noted in the 15-39-year-old subgroup. There were 252 women and 375 men. The median tumor size was $70 \mathrm{~mm}$ (range, $5-950 \mathrm{~mm}$ ) and the median tumor extension was $400 \mathrm{~mm}$ (range, 100-820 $\mathrm{mm}$ ). With regard to the primary tumor site, the distribution was as follows: Head/face/neck (8.0\%), limbs $(48.8 \%)$, thorax/abdomen $(19.9 \%)$ and trunk $(23.3 \%)$. The ICD.O.3 histology groups were adamantinoma of long bones $(4.5 \%)$ and Ewing sarcoma $(95.5 \%)$. In view of the limited number of AJCC T3 $(0.8 \%)$ cases, the main comparison $\chi^{2}$ test was only performed in the AJCC T1 and AJCC T2 cases (53.1 vs. $46.1 \%$ ). AJCC $\mathrm{T}$ was the only variable to do this filtration in the $\chi^{2}$ test, and T3 cases were not deleted in all subsequent analysis processes. Surgery was performed in $76.6 \%$ of patients, with chemotherapy recorded in $92.0 \%$ of the cases, whereas radiation was noted in $40.7 \%$ of the cases. The median OS was 40.00 months (range, 0.00-119.00 months).

Univariate analysis. The K-M survival analysis for OS and CSS is summarized in Table I. Patients who were $>39$ years exhibited the poorest OS and CSS, whereas patients whose age was $<15$ years exhibited the best prognosis $(\mathrm{P}<0.001$, Fig. $2 \mathrm{~A}$; $\mathrm{P}<0.001$, Fig. 2B; P-values apply to the log-rank tests among all categories, not the comparison between any two categories). Improved OS/CSS was achieved in patients who underwent surgery compared with patients who did not undergo surgery $(\mathrm{P}<0.001$, Fig. $2 \mathrm{C} ; \mathrm{P}<0.001$, Fig. 2D). In addition, the MDG values of the random forests were estimated for OS [out of bag $(\mathrm{OOB})=21.37 \%$ ] and $\mathrm{CSS}(\mathrm{OOB}=19.62 \%)$, along with the outcomes of the parametric or the non-parametric tests (Table I). Variance homogeneous and normal distributed continuous variables could be compared by Student's t-test, otherwise, the Mann-Whitney U-test or Kruskal-Wallis H-test 


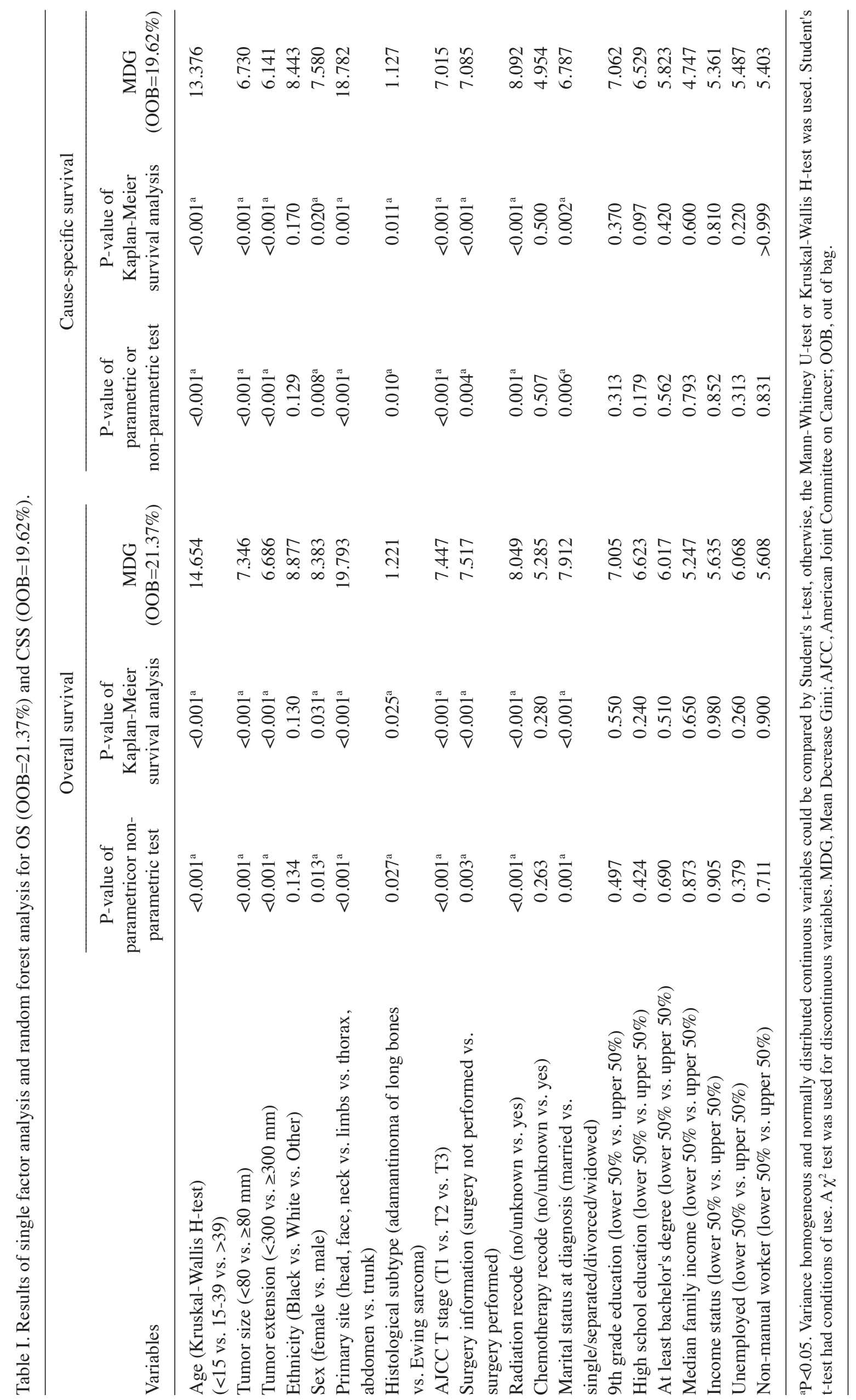


Table II. Cox proportional hazards regression model for overall survival and cause-specific survival in patients with non-metastatic Ewing sarcoma.

\begin{tabular}{|c|c|c|c|c|}
\hline \multirow[b]{2}{*}{ Variable } & \multicolumn{2}{|c|}{ Overall survival } & \multicolumn{2}{|c|}{ Cause-specific survival } \\
\hline & Hazard ratio $(95 \%$ CI) & P-value & Hazard ratio $(95 \% \mathrm{CI})$ & P-value \\
\hline \multicolumn{5}{|l|}{ Categorical age, years } \\
\hline$<15$ & 1.00 (reference) & & 1.00 (reference) & \\
\hline $15-39$ & $2.154(1.375-3.375)$ & $<0.001^{\mathrm{a}}$ & $2.084(1.316-3.300)$ & $0.002^{\mathrm{a}}$ \\
\hline$>39$ & $4.334(2.417-7.770)$ & $<0.001^{\mathrm{a}}$ & $4.121(2.248-7.552)$ & $<0.001^{\mathrm{a}}$ \\
\hline \multicolumn{5}{|l|}{ Categorical tumor extension, $\mathrm{mm}$} \\
\hline$<300$ & 1.00 (reference) & & 1.00 (reference) & \\
\hline$\geq 300$ & $2.404(1.366-4.230)$ & $0.002^{\mathrm{a}}$ & $2.652(1.465-4.803)$ & $0.001^{\mathrm{a}}$ \\
\hline \multicolumn{5}{|l|}{ Sex } \\
\hline Female & 1.00 (reference) & & 1.00 (reference) & \\
\hline Male & $1.654(1.124-2.434)$ & $0.011^{\mathrm{a}}$ & $1.675(1.120-2.506)$ & $0.012^{\mathrm{a}}$ \\
\hline \multicolumn{5}{|l|}{ Histological subtype } \\
\hline Adamantinoma of long bones & 1.00 (reference) & & & \\
\hline Ewing sarcoma & $19.137(2.466-148.492)$ & $0.005^{\mathrm{a}}$ & & \\
\hline \multicolumn{5}{|l|}{ Surgery information } \\
\hline Surgery not performed & 1.00 (reference) & & 1.00 (reference) & \\
\hline Surgery performed & $0.593(0.407-0.865)$ & $0.007^{\mathrm{a}}$ & $0.561(0.378-0.831)$ & $0.004^{\mathrm{a}}$ \\
\hline \multicolumn{5}{|l|}{ Chemotherapy recode } \\
\hline No/Unknown & 1.00 (reference) & & 1.00 (reference) & \\
\hline Yes & $0.308(0.164-0.581)$ & $<0.001^{\mathrm{a}}$ & $0.780(0.400-1.521)$ & 0.466 \\
\hline
\end{tabular}

${ }^{\mathrm{a}}<0.05$.
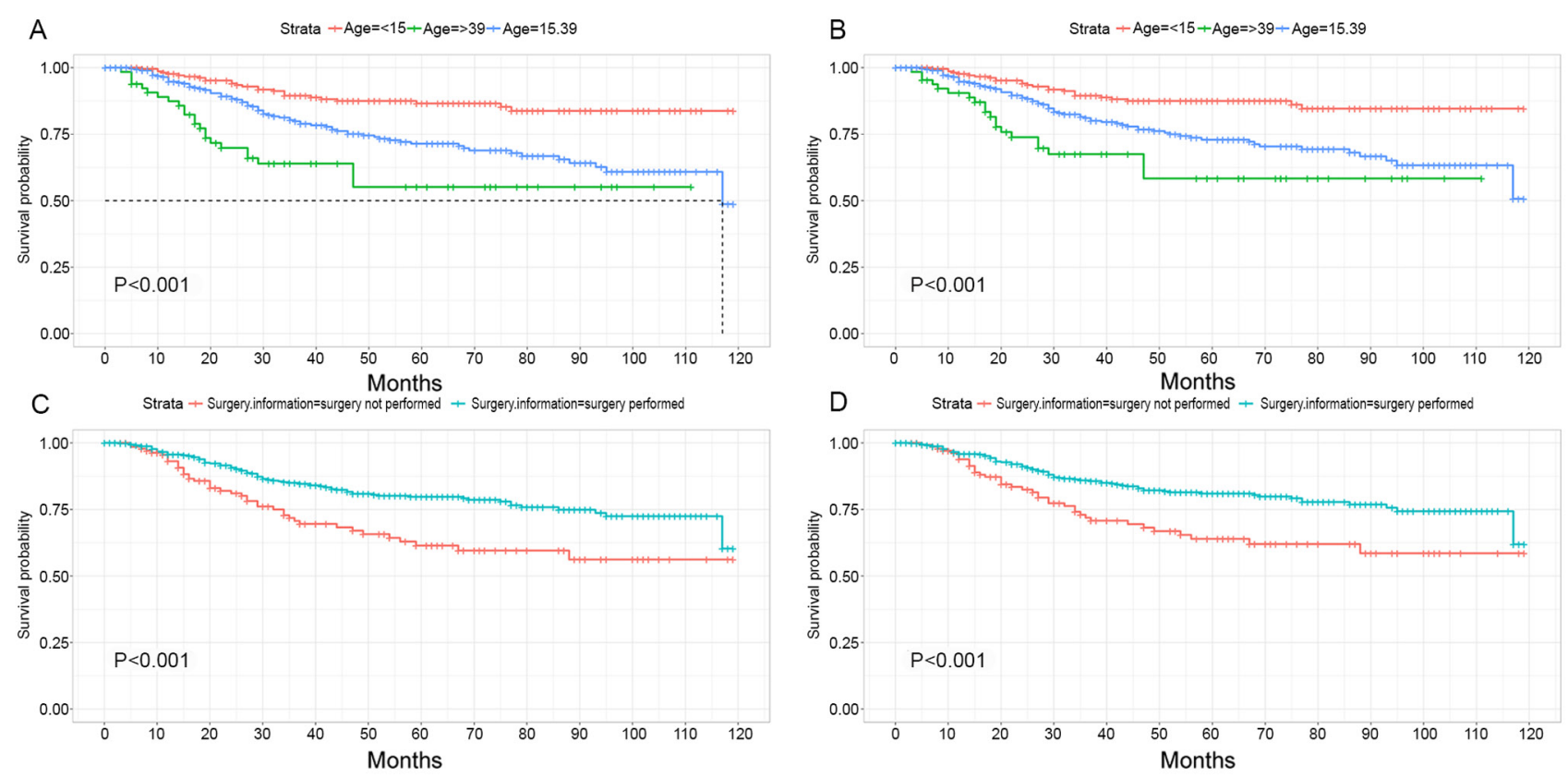

Figure 2. Kaplan-Meier curves of (A and C) overall survival and (B and D) cause-specific survival for (A and B) age and (C and D) surgery.

should be used. The Student's t-test had conditions of use. The $\mathrm{c}^{2}$ test was used for discontinuous variables. In Table II, tumor features and medical treatments were significantly associated with OS and CSS, with the exception of the chemotherapy recode. Chemotherapy recode was also associated with the survival ending (the endpoint of the present study was patient death, which was presented as OS and CSS, and the follow-up endpoint of all patients was December 31, 2014). 
A

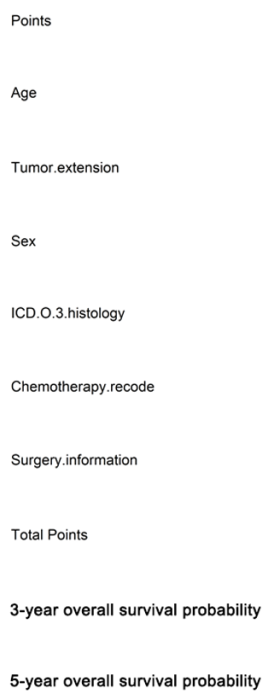

5-year overall survival probability
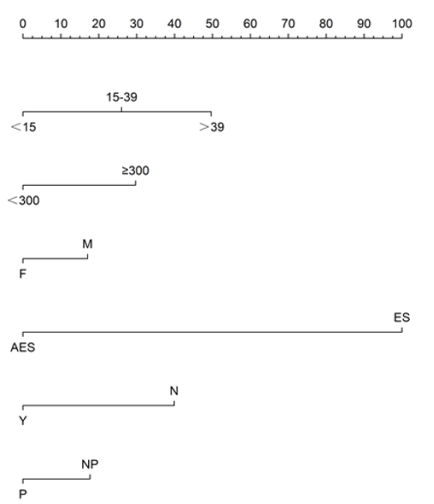

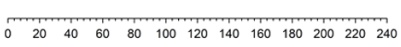

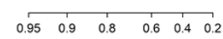

$\begin{array}{lllllll}0.95 & 0.9 & 0.8 & 0.6 & 0.4 & 02\end{array}$

\section{B}

Points

Age

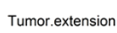

Sex

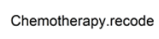

Surgery.information

Total Points

3-year cause-specific survival probability

5-year cause-specific survival probability
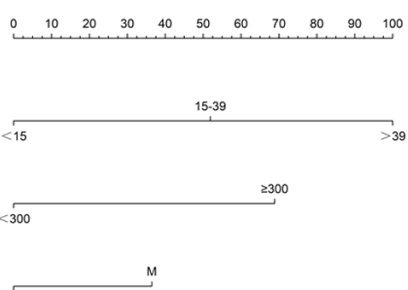

$\stackrel{\text { M }}{u^{\prime}}$
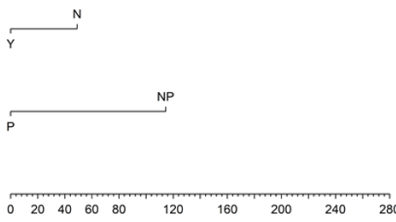

$\begin{array}{rrrrr}0.95 \quad 0.9 & 0.8 & 0.6 & 0.4\end{array}$

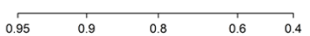

C

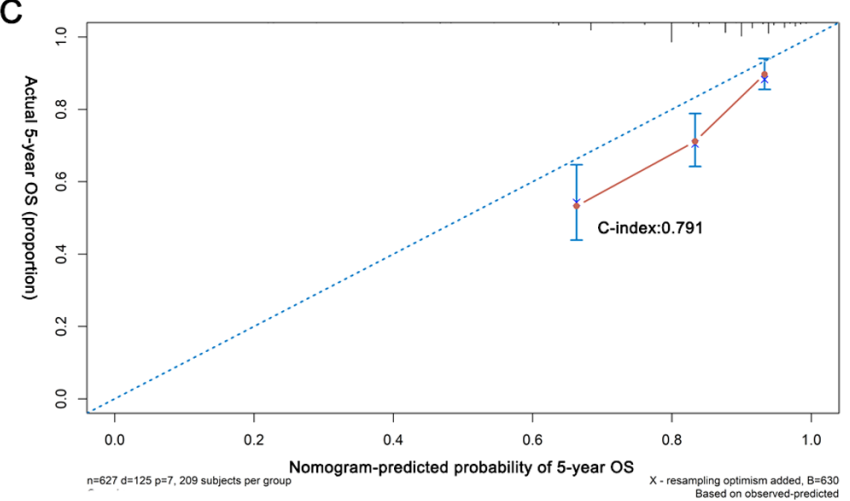

D

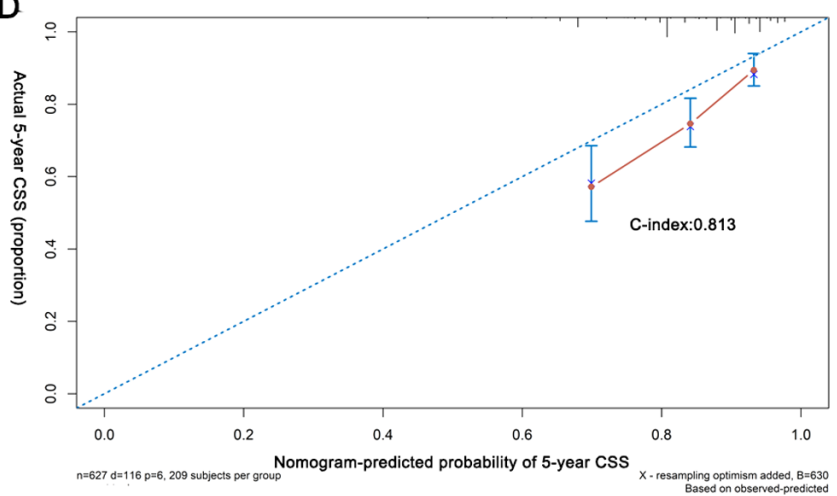

Figure 3. Nomograms and calibration curves of (A and C) OS and (B and D) CSS. The C-indices for internal validation of OS and CSS prediction were 0.791 and 0.813. F, female; M, male; AES, adamantinoma of long bones; ICD.O.3, International Classification of Diseases for Oncology, 3rd Edition; ES, Ewing sarcoma; Y, yes; N, no/unknown; P, surgery performed; NP, surgery not performed; C-index, concordance index; OS, overall survival; CSS, cause-specific survival.

The parameters age, sex and marital status (data not shown) significantly influenced the prognosis of the patients. Finally, 11 elements (age, sex, tumor size, tumor extension, primary site, ICD.O.3 histology, AJCC T, surgery information, radiation recode, chemotherapy recode and marital status) were further analyzed using the Cox regression model.

Multivariate Cox regression and nomogram. In the Cox proportional hazards model, a higher hazard ratio (HR) was noted for the parameter of older age for OS [15-39 years: HR, 2.154; 95\% confidence interval (CI), 1.375-3.375; $\mathrm{P}<0.001$; $>39$ years: HR, 4.334; 95\% CI, 2.417-7.770; $\mathrm{P}<0.001]$ and CSS (15-39 years: HR, 2.084; 95\% CI, 1.316-3.300; $\mathrm{P}=0.002$; $>39$ years: $\mathrm{HR}, 4.121 ; 95 \%$ CI, 2.248-7.552; $\mathrm{P}<0.001)$ compared with younger age $(<15$ years). Larger tumor extension was also a risk factor for OS ( $\geq 300.00 \mathrm{~mm}$ : HR, 2.404; 95\% CI, 1.366-4.230; $\mathrm{P}=0.002)$ and CSS $(\geq 300.00 \mathrm{~mm}$ : HR, 2.652; 95\% CI, 1.465-4.803; $\mathrm{P}=0.001)$. In addition, male subjects appeared to have poorer OS (HR, 1.654; 95\% CI, 1.124-2.434; $\mathrm{P}=0.011)$ and CSS (HR, 1.675; 95\% CI, 1.120-2.506; $\mathrm{P}=0.012$ ) than female subjects. The ICD.O.3 histological results for the higher risk subtype (Ewing sarcoma) indicated associations with OS (HR, 19.137; 95\% CI, 2.466-148.492; P=0.005), but not with CSS (data not shown). The patients who had undergone surgery exhibited favorable OS (HR, 0.593; 95\% CI, 0.407-0.865; $\mathrm{P}=0.007)$ and CSS (HR, 0.561; 95\% CI, $0.378-0.831 ; \mathrm{P}=0.004)$. The patients who were treated with chemotherapy exhibited improved OS (HR, 0.308; 95\% CI, $0.164-0.581 ; \mathrm{P}<0.001)$, whereas the effects of chemotherapy on CSS (HR, 0.780; 95\% CI, 0.400-1.521; P=0.466) were not identified to be statistically significant.

Based on the reduced Cox models, nomograms were built to predict the probability of 3- and 5-year OS (Fig. 3A and C; C-index=0.791) and CSS (Fig. 3B and D; C-index=0.813). The calibration plots demonstrated the agreement between predictions and observed values due to the 45-degree-line nearby points (Fig. 3C and D). Information of the 26 cases for external validation is shown in Table SIII and the median age was 18 years (range, 1-51 years). The median survival time was 22 months (range, 3-54 months; Table SIII). The concordances of external validation based on the cohort of The First Affiliated Hospital of Zhengzhou University (Zhengzhou, 
China) were 0.834 for OS and 0.825 for CSS. Although the results of the K-M survival analysis indicated significant prognostic values of AJCC stage, the C-indices were 0.531 (OS) and 0.534 (CSS) for AJCC 6th edition guidelines, and 0.547 (OS) and 0.561 (CSS) for AJCC 7th edition guidelines, respectively, which were lower than the nomograms (Fig. S1). Additionally, the results of the K-M survival analysis indicated that chemotherapy could not significantly prolong the survival of the patients (Fig. S2). In the subgroup analysis, older patients were more likely to undergo chemotherapy rather than surgery, which might explain the poor prognosis in older patients (Table SIV).

\section{Discussion}

Ewing sarcoma is the second most common malignant osseous tumor occurring in children and adolescents after osteosarcoma (2). Non-metastatic Ewing sarcoma has a favorable prognosis compared with the metastatic type and the identification of its predictors is helpful to prolong OS and CSS (6). A nomogram is a widely used prediction model that can incorporate meaningful factors, and predict OS and CSS of patients. In the present study, a prognostic nomogram was constructed for patients with non-metastatic Ewing sarcoma based on the SEER database. Furthermore, external non-metastatic Ewing sarcoma cases from The First Affiliated Hospital of Zhengzhou University (Zhengzhou, China) were used to validate the nomograms in order to provide reliable prediction models for clinical treatment.

The machine learning model (random forest) and classical survival analysis (K-M survival curve and Cox proportional hazards regression model) demonstrated that age, sex, tumor extension and ICD.O.3 histology were independent prognostic factors for both OS and CSS. Surgery was identified as a favorable predictor for both OS and CSS, while chemotherapy was significantly associated with OS and could potentially influence CSS in a number of previous studies, but this was not a significant indicator for CSS in the present study $(6,8,17,18)$. Other variables, namely ethnicity, tumor size, primary site, radiotherapy and socioeconomic status (marital status), were not observed to be independent risk factors associated with OS and CSS.

In the present series of patients, the mean age was 20.14 \pm 13.62 years, with 375 male and 252 female subjects. Old age and male sex were prognostic factors for OS and CSS as determined by univariate and multivariate analyses, which was in accordance with previously reported findings (2,18-24). In the subgroup analysis, older patients were more likely to undergo chemotherapy rather than surgery, which might explain the poor prognosis in older patients (Table SIV). Therefore, it was suspected that the poor prognosis of older patients may be due to their low tolerance of chemotherapy $(25,26)$. Furthermore, the results indicated that male patients tended to develop large tumors, which may increase the treatment complexity (Table SIV).

Ewing sarcoma was divided into adamantinoma-like Ewing sarcoma of long bones and Ewing sarcoma based on its histological evaluation provided by the SEER database. The percentages of the incidence of these tumors were 4.5 and $95.5 \%$, respectively. Due to the lower-grade malignancy of adamantinoma-like Ewing sarcoma, ICD.O.3 histology was a significant variable in the current analysis, and Ewing sarcoma exhibited a poorer prognosis than the adamantinoma of long bones $(27,28)$.

The tumor extension was an independent prognostic factor for both OS and CSS in the present analysis. Based on the results of the subgroup analysis, larger extension limited the application of surgery, thereby leading to negative prognosis, which was similar to the findings reported by Arshi et al (2).

Current therapeutic strategies for Ewing sarcoma include surgery, chemotherapy and radiotherapy, and their combined application. Surgery is the standard treatment strategy for Ewing sarcoma $(7,12,23,24)$. In the present series, $76.6 \%$ of patients underwent surgery and this treatment method was a favorable prognostic factor for both OS and CSS. With regard to non-metastatic Ewing sarcoma, surgery is more useful due to the high probability of en bloc tumor resection $(12,17,18)$. Chemotherapy is the main treatment method for Ewing sarcoma, which was present in $\sim 92 \%$ of the cases comprising non-metastatic Ewing sarcoma in the present study $(7,12)$. Additionally, chemotherapy has been demonstrated to be a favorable predictor for OS (7,12). In Europe, the standard chemotherapeutic strategy for Ewing sarcoma is vincristine, ifosfamide, doxorubicin and etoposide (VIDE). This regimen is safely used at a high dose and is administered bi-weekly $(5,29)$. It destroys any remaining tumor cells that are proliferating slowly, and further improves patient survival time (1). In addition, in the external validation cohort of patients in the present study, the chemotherapeutic regimen used was a VIDE regimen in all cases. The results of the K-M survival analysis indicated that chemotherapy could significantly prolong the survival of the patients. Furthermore, radiotherapy has been reported to be an appropriate regimen for local control in patients with Ewing sarcoma (13). In the present study, non-significant results were noted in the Cox model, which was consistent with Gaspar et al (5), who demonstrated that radiotherapy did not exhibit a long-term tumor control and may result in severe side effects.

Based on the six independent variables identified using univariate and multivariate analyses, nomograms with high AUC and collaboration were constructed for OS and CSS in order to predict patient survival probability at certain time points. By external validation, they were appropriately tested and achieved satisfying fitting degrees. The C-index was estimated to be 0.931 and 0.724 for OS and CSS, respectively, which was superior to those for the AJCC 6th and 7th editions. Therefore, this variable could assist clinicians in making accurate survival evaluations and generating appropriate therapeutic strategies.

To the best of our knowledge, the present study was the first to address the use of nomograms in the field of non-metastatic Ewing sarcoma. The results implied the potential for clinical application. Despite these promising results, several limitations were present in the current study. Inaccurate variables appeared due to the criteria discrepancy in diagnosis and treatment methods that originated from different registries. Secondly, the retrospective nature of the present study was a limitation compared with a prospective study, such as a prospective cohort study and randomized controlled trial. Thirdly, the study lacked the detailed information for the chemotherapy and radiotherapy protocols used in the SEER database, including the 
treatment time, dose cycle, drug type and dosage (30). Finally, although applicable nomograms for OS and CSS have been tested by internal and external validation protocols alongside the calibration curves, a limited number of cases in the external series had a relatively short follow-up time. Additional clinical data are required to validate and revise the data presented in the current study. In order to further analyze and improve the application of nomograms, more strict and accurate models that can combine the variables examined with genetic factors are required. Subsequent studies should focus on the complex molecular mechanisms and expression of genomic biomarkers in order to construct more accurate nomograms.

In conclusion, age, sex, tumor extension and surgery were independent prognostic factors for both OS and CSS. In addition, the Ewing sarcoma subtype was a poor factor, whereas chemotherapy was a favorable factor with regard to OS. Nomograms based on reduced Cox models attained a satisfying accuracy in predicting the survival of patients with non-metastatic Ewing sarcoma and could assist clinicians in more accurately evaluating patient survival.

\section{Acknowledgements}

Not applicable.

\section{Funding}

The present study was supported in part by the National Natural Science Foundation of China (grant nos. 81702659, 81772856 and 81501203), Youth Fund of Shanghai Municipal Health Planning Commission (grant no. 2017YQ054) and Henan Medical Science and Technology Research Project (grant no. 201602031).

\section{Availability of data and materials}

All data generated or analyzed during this study are included in this published article.

\section{Authors' contributions}

RH, DH, CS, PY, PH, XZ, HY, TM and ZH conceived and designed the study, collected and/or assembled the data. analyzed and interpreted the data and wrote the manuscript. RH, DH, CS, PY, PH, XZ, HY, TM and ZH confirmed the authenticity of all the raw data. All authors read and approved the final manuscript.

\section{Ethics approval and consent to participate}

The present study was approved by the Ethics Committee of The First Affiliated Hospital of Zhengzhou University (approval no. KEYAN-2018-LW-023; Zhengzhou, China). All patients enrolled in the present study provided written informed consent for participation.

\section{Patient consent for publication}

Written informed consent was obtained from all 26 patients for publication of the present study. Consent was obtained from the parents/guardians of the patients under 18 years of age at the time of data collection.

\section{Competing interests}

The authors declare that they have no competing interests.

\section{References}

1. Grunewald TGP, Cidre-Aranaz F, Surdez D, Tomazou EM, de Álava E, Kovar H, Sorensen PH, Delattre O and Dirksen U: Ewing sarcoma. Nat Rev Dis Primers 4: 5, 2018.

2. Arshi A, Sharim J, Park DY, Park HY, Yazdanshenas H, Bernthal NM and Shamie AN: Prognostic determinants and treatment outcomes analysis of osteosarcoma and Ewing sarcoma of the spine. Spine J 17: 645-655, 2017.

3. Scotlandi K, Baldini N, Cerisano V, Manara MC, Benini S, Serra M, Lollini PL, Nanni P, Nicoletti G, Bernard G, et al: CD99 engagement: An effective therapeutic strategy for Ewing tumors. Cancer Res 60: 5134-5142, 2000.

4. Riggi N, Cironi L, Provero P, Suvà ML, Kaloulis K, GarciaEcheverria C, Hoffmann F, Trumpp A and Stamenkovic I: Development of Ewing's sarcoma from primary bone marrow-derived mesenchymal progenitor cells. Cancer Res 65: $11459-11468,2005$.

5. Gaspar N, Hawkins DS, Dirksen U, Lewis IJ, Ferrari S, Le Deley MC, Kovar H, Grimer R, Whelan J, Claude L, et al: Ewing sarcoma: Current management and future approaches through collaboration. J Clin Oncol 33: 3036-3046, 2015.

6. Biermann JS, Chow W, Reed DR, Lucas D, Adkins DR, Agulnik M, Benjamin RS, Brigman B, Budd GT, Curry WT, et al: NCCN Guidelines Insights: Bone cancer, version 2.2017. J Natl Compr Canc Netw 15: 155-167, 2017.

7. Arpaci E, Yetisyigit T, Seker M, Uncu D, Uyeturk U, Oksuzoglu B, Demirci U, Coskun U, Kucukoner M, Isıkdogan A, et al: Prognostic factors and clinical outcome of patients with Ewing's sarcoma family of tumors in adults: Multicentric study of the Anatolian Society of Medical Oncology. Med Oncol 30: 469, 2013.

8. Paulussen M, Ahrens S, Burdach S, Craft A, DockhornDworniczak B, Dunst J, Fröhlich B, Winkelmann W, Zoubek A and Jürgens H: Primary metastatic (stage IV) Ewing tumor: Survival analysis of 171 patients from the EICESS studies. European Intergroup Cooperative Ewing Sarcoma Studies. Ann Oncol 9: 275-281, 1998

9. Ferreira AJ, Boldrini E, López RVM, Scapulatempo Neto C, Santos JF and Lopes LF: Characterization, survival analysis, and expression of IGFR in tumor samples from patients diagnosed with Ewing family tumors treated at the Barretos Cancer Hospital. Rev Bras Ortop 52: 705-713, 2016.

10. Rodríguez-Galindo C, Liu T, Krasin MJ, Wu J, Billups CA, Daw NC, Spunt SL, Rao BN, Santana VM and Navid F: Analysis of prognostic factors in ewing sarcoma family of tumors: Review of St. Jude Children's Research Hospital studies. Cancer 110: 375-384, 2007.

11. Grevener K, Haveman LM, Ranft A, van den Berg H, Jung S, Ladenstein R, Klco-Brosius S, Juergens H, Merks JH and Dirksen U: ManagementandoutcomeofEwingsarcomaoftheheadandneck.Pediatr Blood Cancer 63: 604-610, 2016.

12. Wan W, Lou Y, Hu Z, Wang T, Li J, Tang Y, Wu Z, Xu L, Yang X, Song D and Xiao J: Factors affecting survival outcomes of patients with non-metastatic Ewing's sarcoma family tumors in the spine: A retrospective analysis of 63 patients in a single center. J Neurooncol 131: 313-320, 2017.

13. Ning MS, Perkins SM, Borinstein SC, Holt GE, Stavas MJ and Shinohara ET: Role of radiation in the treatment of non-metastatic osseous Ewing sarcoma. J Med Imaging Radiat Oncol 60: 119-128, 2016.

14. Buja A, Lago L, Lago S, Vinelli A, Zanardo C and Baldo V: Marital status and stage of cancer at diagnosis: A systematic review. Eur J Cancer Care (Engl) 27, 2018.

15. Gomez SL, Hurley S, Canchola AJ, Keegan TH, Cheng I, Murphy JD, Clarke CA, Glaser SL and Martínez ME: Effects of marital status and economic resources on survival after cancer: A population-based study. Cancer 122: 1618-1625, 2016.

16. Niu XH. Interpretation of 2020 NCCN Clinical Practice Guidelines in Oncology-Bone Cancer. Zhonghua Wai Ke Za Zhi. 58: 430-434, 2020 (In Chinese). 
17. Coccia PF, Pappo AS, Beaupin L, Borges VF, Borinstein SC, Chugh R, Dinner S, Folbrecht J, Frazier AL, Goldsby R, et al: Adolescent and young adult oncology, version 2.2018. J Natl Compr Cancer Netw 16: 66-97, 2018.

18. Bacci G, Longhi A, Ferrari S, Mercuri M, Versari M and Bertoni F: Prognostic factors in non-metastatic Ewing's sarcoma tumor of bone: An analysis of 579 patients treated at a single institution with adjuvant or neoadjuvant chemotherapy between 1972 and 1998. Acta Oncol 45: 469-475, 2006.

19. Jawad MU, Cheung MC, Min ES, Schneiderbauer MM Koniaris LG and Scully SP: Ewing sarcoma demonstrates racial disparities in incidence-related and sex-related differences in outcome: An analysis of 1631 cases from the SEER database, 1973-2005. Cancer 115: 3526-3536, 2009.

20. Duchman KR, Gao Y and Miller BJ: Prognostic factors for survival in patients with Ewing's sarcoma using the surveillance, epidemiology, and end results (SEER) program database. Cancer Epidemiol 39: 189-195, 2015.

21. Karski EE, Matthay KK, Neuhaus JM, Goldsby RE and Dubois SG: Characteristics and outcomes of patients with Ewing sarcoma over 40 years of age at diagnosis. Cancer Epidemiol 37: 29-33, 2013.

22. López Guerra JL, Márquez-Vega C, Ramírez-Villar GL, Cabrera P, Ordóñez R, Praena-Fernández JM and Ortiz MJ: Prognostic factors for overall survival in paediatric patients with Ewing sarcoma of bone treated according to multidisciplinary protocol. Clin Transl Oncol 14: 294-301, 2012.

23. Oksüz DC, Tural D, Dincbas FÖ, Dervisoglu S, Turna H, Hiz M, Kantarci F, Ceylaner B, Koca S and Mandel NM: Non-metastatic Ewing's sarcoma family of tumors of bone in adolescents and adults: Prognostic factors and clinical outcome-single institution results. Tumori 100: 452-458, 2014.

24. WanZH,Huang ZH and Chen LB: Survival outcome among patients with Ewing's sarcoma of bones and joints: A population-based cohort study. Sao Paulo Med J 136: 116-122, 2017.
25. Gupta AA,Pappo A, Saunders N, Hopyan S, Ferguson P, Wunder J, O'Sullivan B, Catton C, Greenberg M and Blackstein M: Clinical outcome of children and adults with localized Ewing sarcoma: Impact of chemotherapy dose and timing of local therapy. Cancer 116: 3189-3194, 2010 .

26. Lee J, Hoang BH, Ziogas A and Zell JA: Analysis of prognostic factors in Ewing sarcoma using a population-based cancer registry. Cancer 116: 1964-1973, 2010.

27. Kreicbergs A, Silvferswärd C and Tribukait B: Flow DNA analysis of primary bone tumors. Relationship between cellular DNA content and histopathologic classification. Cancer 53: 129-136, 1984.

28. Fujii H, Honoki K, Enomoto Y, Kasai T, Kido A, Amano I, Kumamoto M, Morishita T, Mii Y, Nonomura A and Takakura Y: Adamantinoma-like Ewing's sarcoma with EWS-FLI1 fusion gene: A case report. Virchows Arch 449: 579-584, 2006.

29. Womer RB, West DC, Krailo MD, Dickman PS, Pawel BR, Grier HE, Marcus K, Sailer S, Healey JH, Dormans JP and Weiss AR: Randomized controlled trial of interval-compressed chemotherapy for the treatment of localized Ewing sarcoma: A report from the Children's Oncology Group. J Clin Oncol 30: 4148-4154, 2012.

30. Noone AM, Lund JL, Mariotto A, Cronin K, McNeel T, Deapen D and Warren JL: Comparison of SEER treatment data with medicare claims. Med Care 54: E55-E64, 2016.

cc) (i) $\Theta$ This work is licensed under a Creative Common International (CC BY-NC-ND 4.0) License. 